\title{
The epidemiology and transmission of methicillin-resistant Staphylococcus aureus in the community in Singapore: study protocol for a longitudinal household study
}

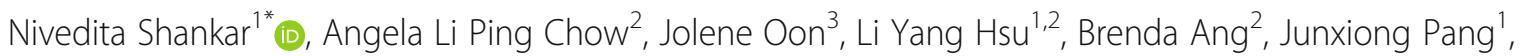
Paola Florez De Sessions ${ }^{4}$, Balamurugan Periaswamy ${ }^{4}$, Paul A. Tambyah ${ }^{3}$, Desmond B. Teo ${ }^{3}$ and Clarence C. Tam ${ }^{1,5}$

\begin{abstract}
Background/aim: Methicillin-resistant Staphylococcus aureus (MRSA) is one of the most common multidrug-resistant organisms in healthcare settings worldwide, but little is known about MRSA transmission outside of acute healthcare settings especially in Asia. We describe the methods for a prospective longitudinal study of MRSA prevalence and transmission.

Methods: MRSA-colonized individuals were identified from MRSA admission screening at two tertiary hospitals and recruited together with their household contacts. Participants submitted self-collected nasal, axilla and groin (NAG) swabs by mail for MRSA culture at baseline and monthly thereafter for 6 months. A comparison group of households of MRSA-negative patients provided swab samples at one time point. In a validation sub-study, separate swabs from each site were collected from randomly selected individuals, to compare MRSA detection rates between swab sites, and between samples collected by participants versus those collected by trained research staff. Information on each participant's demographic information, medical status and medical history, past healthcare facilities usage and contacts, and personal interactions with others were collected using a self-administered questionnaire.

Discussion/conclusion: Understanding the dynamics of MRSA persistence and transmission in the community is crucial to devising and evaluating successful MRSA control strategies. Close contact with MRSA colonized patients may to be important for MRSA persistence in the community; evidence from this study on the extent of community MRSA could inform the development of household- or community-based interventions to reduce MRSA colonization of close contacts and subsequent re-introduction of MRSA into healthcare settings. Analysis of longitudinal data using whole-genome sequencing will yield further information regarding MRSA transmission within households, with significant implications for MRSA infection control outside acute hospital settings.
\end{abstract}

Keywords: Cohort studies, Longitudinal studies, MRSA, Staphylococcus aureus, Methicillin, Antimicrobial resistance epidemiology, Community, Colonization, Household transmission

\footnotetext{
* Correspondence: ephns@nus.edu.sg

${ }^{1}$ Saw Swee Hock School of Public Health, Tahir Foundation Building, 12

Science Drive 2, 11-01, Singapore 117549, Singapore

Full list of author information is available at the end of the article
} 


\section{Background}

Since its emergence in the 1960s, methicillin-resistant Staphylococcus aureus (MRSA) has become one of the most common multidrug-resistant organisms in healthcare settings worldwide, resulting in considerable health and economic burden. Carriage of MRSA is a major risk factor for invasive MRSA infection [1-8], and patient transfers between acute care hospitals, community hospitals and longterm care facilities have been reported to be important for maintaining MRSA transmission in healthcare settings [917]. MRSA transmission from MRSA-infected patients and MRSA carriers to their household contacts has also been documented [18-22], but the importance of household transmission for persistence of MRSA in the community and reintroduction into healthcare settings is not well understood. A number of cross-sectional studies have suggested that between $20 \%$ and $30 \%$ of household contacts of MRSA positive index patients are colonized with MRSA [23, 24]. A limited number of longitudinal studies have shown that MRSA transmission to household contacts is common, with $20 \%-40 \%$ of non-colonized household contacts of MRSA positive individuals acquiring MRSA during the follow-up period [18, 20-22]. Identified risk factors for MRSA acquisition among household contacts include older age [20] and providing care to MRSA-colonized individuals [20, 25].

In Singapore, MRSA is endemic in healthcare facilities $[26,27]$. A laboratory-based program in six public sector acute care hospitals (ACHs) reported that $35 \%$ of all clinical S. aureus isolates were methicillin-resistant [26]. MRSA Sequence Type (ST) 239 was predominant in Singaporean hospitals in the mid-1980s, but was rapidly supplanted as the major clone following the introduction of ST22 in the early 2000s [28]. More recently, ST45 was introduced into local hospitals. Community-associated (CA) MRSA strains have also been identified sporadically since the early 2000s, with ST30 being the predominant clonal strain [29].

Specific measures adopted by healthcare institutions to control MRSA include active surveillance in tertiary care hospitals, cohorting or isolation of MRSA colonized patients and hand hygiene initiatives. Historical evaluations of these bundled MRSA interventions at two major tertiary care hospitals have documented a sustained decrease in MRSA infection and hospital acquired bacteraemia rates of $50 \%$ - $60 \%$ between 2004 and 2012, alongside significant increases in hand hygiene compliance among healthcare workers [30]. Reducing colonization is believed to be crucial to reducing MRSA burden, as colonization is a major risk factor for subsequent infection, and infection carries a high risk of excess morbidity and mortality [25, 26, 31, 32].

Despite these efforts to control MRSA transmission in $\mathrm{ACHs}$, intermediate-term and long-term care facilities remain as important reservoirs [11]. A recent survey indicated that MRSA colonization prevalence is substantially higher among patients in intermediate-term (29.9\%) and long-term $(20.4 \%)$ care facilities compared with acute care hospitals (11.8\%) [11]. Several studies have suggested a role of long-term care facilities for sustaining MRSA endemicity in healthcare networks $[11,14,16,17,33]$. However, relatively little is known about the role of MRSA transmission in the wider, non-hospitalized community in maintaining MRSA transmission and reintroducing MRSA strains into healthcare settings. A few longitudinal studies have demonstrated the potential for MRSA transmission from colonized patients to their household contacts, suggesting the existence of a large reservoir of MRSA in the general population. However, the relationship between these community transmission events and the epidemiology of MRSA in healthcare settings is not well understood.

In recent years, whole genome sequencing (WGS) has emerged as the leading technology for the study of bacterial population genetics and transmission dynamics [34-37]. WGS has provided crucial insights into the epidemiology of antibiotic resistance, the genetics of virulence, the origins and expansion of lineages, and the population structure of $S$. aureus [38]. The high resolution of WGS means that it offers superior data compared with other molecular typing techniques to determine transmission events, and profile resistance, virulence and even the type isolates [38-40] within days [41]. Various studies have used WGS to track MRSA transmission networks in healthcare settings [9, 11, 12, 33, 42-44] and the community [44]. To our knowledge, however, no study has investigated MRSA carriage and transmission among households using WGS. We describe the methods for an ongoing longitudinal study to investigate the persistence and transmissibility of MRSA in the community, and to quantify the relationship between healthcare contact and community transmission.

\section{Methods}

The overall aim of this study is to determine the dynamics of MRSA carriage, persistence and transmission in the community. The specific objectives are:

a) To measure frequency of MRSA colonization among household contacts

b) To identify risk factors associated with colonization and prolonged carriage

c) To study MRSA transmission in household contacts of MRSA-colonized patients (after discharge)

d) To identify factors associated with MRSA persistence in the household

e) To quantify importance of healthcare contact, and other personal interactions, in the transmission of MRSA in the community

f) To determine the diversity of MRSA strains 
This study will comprise a baseline survey of MRSA prevalence and risk factors among household contacts of 100 MRSA-colonized and 100 MRSA non-colonized index patients, followed by a 6-month longitudinal study among MRSA-colonized index patients and their household contacts, with monthly self-swabbing and questionnaire interview.

Index patients will be recruited from two acute and tertiary care hospitals in Singapore. Hospital 1 has 1600 beds and primarily serves an elderly population. Active MRSA admission screening based on nasal swab polymerase chain reaction (PCR) has been conducted since 2009. Positive patients are cohorted in dedicated MRSA wards. Hospital 2 is a university hospital with 1000 beds serving a mixed population. Active MRSA admission screening based on nose, axilla and groin swab culture has been conducted since 2010. Positive patients are allocated to dedicated MRSA cubicles within each ward.

Potential index patients for the MRSA-colonized and MRSA non-colonized arms will be selected from daily lists of patients undergoing MRSA screening on admission. Index patients will be eligible for inclusion in the MRSA-colonized arm if:

1) They are aged 21 years or above

2) They have a positive MRSA screen at admission

3) They live in a household with at least one other person who routinely spends nights at the same address

Patients will be excluded if they live in an institutionalized setting, such as a nursing home, or if they are admitted to an intensive care unit at the time of recruitment. Eligibility and exclusion criteria for index patients in the MRSA non-colonized arm will be the same, with the exception that the patient must test negative for MRSA upon admission screening.

Eligible patients will be approached in the ward by trained research staff to explain the study in the patients' preferred language (English, Mandarin, Malay or Tamil) and to invite them to participate in the study. Interested patients will be asked to provide signed informed consent and consent to contact their household for the purposes of recruiting household members. For potential participants suffering from cognitive impairment, informed consent will be obtained from their legally appointed representative, usually a spouse or family member responsible for making healthcare decisions on behalf of their patients.

Research staff will then arrange a house visit to recruit household contacts of the index case. A household contact will be defined as a person who routinely spends nights at the same address as the index patient. Household contacts interested in taking part will be asked to provide signed informed consent. Parental consent and participant assent will be obtained for participants aged six to 20 years; children below six years will not be eligible to participate. A baseline questionnaire will be administered to each participating household member, to collect information on each participant's demographic information, medical status and medical history, past healthcare facilities usage and contacts, and personal interactions with others. Participants will then be shown the correct procedure for obtaining swabs from the nares, axilla and groin $(\mathrm{N}, \mathrm{A}, \mathrm{G})$, and asked to provide an $\mathrm{N}$ and combined AG swab using a Copan E-swab MRSA Collection System (Copan Diagnostics).

For participants in the MRSA-colonized arm taking part in the follow-up component, written instructions and links to instructional videos for the self-swabbing procedure will be provided for future reference. Participants will be instructed to take two swabs, one from the nares and a second from the axillary and groin areas, and combine them in a single sample tube. Colourcoded stickers will be pre-labelled with participants' identification number and used to label the sample tubes. Pre-paid, addressed, padded envelopes will be provided for participants to send the swabs directly to the laboratory. Participants will submit swabs two and four weeks after enrolment and at monthly intervals thereafter, at a pre-specified time of the month, for a period of 6 months. Reminders will be sent by SMS. After 6 months, a second household visit will be arranged to update information on healthcare contacts in the previous 6 months, recent travel history and contact patterns.

In a validation sub-study, the research team will take swabs of the nares and axilla from a randomly selected household member at the initial recruitment visit. The selected household member will then take repeat swabs by themselves, and take a third swab from the groin area in a separate room. The five swab samples will be collected in separate sample tubes, using the Copan E-swab MRSA Collection System, and cultured individually to validate the self-swabbing procedure to be used for MRSA testing during the follow-up period.

Receipt of swab specimens will be recorded at the laboratory located near hospital 1. A study team member will liaise with the laboratory to ensure that specimens have been received. Households from which samples have not been received by the pre-specified date will be contacted by the research nurse to ensure that samples are taken and sent to the laboratory.

\section{Sample size}

Given an average household size of three persons, we anticipate 200 household contacts in each arm. Lucet et al. reported $88 \%$ participation among household contacts $^{16}$. Assuming 80\% participation among household contacts, a study with 160 participants in each arm, for a 
total sample size of 320 , will enable us to detect a minimum of $13 \%$ difference in MRSA prevalence between the two groups. These estimates assume a Type I error probability of $0.05,80 \%$ power and a design effect (DEFF) of 1.25 resulting from the clustered nature of the survey design. We expect the design effect to be minimal, because of the small average number of participants recruited per household.

\section{Laboratory procedures}

All swabs will be tested for MRSA by culture on selective chromogenic media (Bio-Rad MRSA Select ${ }^{\mathrm{m}}$ ), followed by a confirmatory agglutination test (Bio-Rad Pastorex Staph Plus). If the first two tests are deemed inconclusive, a third coagulase test will be conducted.

Swab samples were plated onto selective chromogenic media using sterile inoculating loops. The plates will be incubated at $35-37{ }^{\circ} \mathrm{C}$ at ambient temperature and read at 18-24 h. Negative plates or plates with minimal growth will be re-incubated and re-read at $48 \mathrm{~h}$. A latex agglutination test will be performed on culture plates with suspected MRSA colonies to confirm the presence of Staphylococcus aureus.

\section{Storage of MRSA isolates}

Using a sterile loop, a mixture of different MRSA colonies will be picked and emulsified in the cryopreservative fluid. If culture growth is scarce, a subculture will be performed and the colonies picked for storage in cryocare vials (Key Scientific Products, Stamford, TX) at $-80^{\circ} \mathrm{C}$.

\section{Whole genome sequencing of MRSA isolates}

The whole genome sequencing phase of the study will require DNA to be extracted from the MRSA isolates. The MRSA isolates from the hospital laboratory are recultured on blood agar plates (BD Diagnostics, USA $\mathrm{BBL}^{\mathrm{Tu}}$ Columbia Agar with 5\% sheep blood) from cryocare vials, and 5 individual colonies are picked per plate for overnight culture in Luria-Bertani (LB) broth (Gibco ${ }^{\circ}$ LB Broth, Liquid). $900 \mu \mathrm{l}$ from each overnight culture is resuspended with $100 \mu \mathrm{l}$ of sterile DMSO and stored as independent MRSA isolates. The remaining DNA will be extracted from these overnight cultures using the QIAamp DNA Mini Kit (Qiagen Inc., Hilden, Germany).

Where multiple transmissions are suspected between household members (for example, where a previously negative household member is subsequently colonized), a subset of strains from serial swabs will undergo whole genome sequencing for molecular epidemiological studies. Genomic DNA will be extracted from S. aureus cultures derived from swab samples of index patients and household members. The isolates will be used to provide background genetic information on the $S$. aureus strains circulating locally. Genomic DNA will be extracted using the QIAamp DNA Mini Kit (Qiagen Inc., Hilden, Germany). DNA shearing will be done using Covaris LE220 Focused-ultrasonicator followed by library preparation using NEBNext ${ }^{\circ}$ Ultra $^{\mathrm{Tm}}$ DNA Library Prep Kit for Illumina ${ }^{\circ}$ (E7370L) and custom primers with 8 bp barcode index. Sequencing will be done on an Illumina HiSeq $4 \mathrm{~K}$ platform.

\section{Ethical approval}

Ethical approval for this study has been obtained from the National Health Group Domain Specific Review Board (NHG-DSRB 2014/01278).

\section{Data analysis}

MRSA test results for duplicate swabs taken by trained researchers, considered a "gold-standard test", and participants themselves from the same site will be used to determine the sensitivity of self-swabbing. Sensitivity will be defined as the proportion of positive nurseadministered swabs for which the paired, selfadministered swab is also positive. Sensitivity will be calculated for MRSA, and separately for nasal and axillary swabs. Sensitivity estimates will be expressed as percentages with corresponding 95\% confidence intervals (CI).

Duration of MRSA carriage, by anatomical site, will be quantified using survival analysis methods. Survival curves will be plotted to show the changes in carriage over time from serial samples. Survival curves will also be used to investigate the incidence of colonization among initially MRSA negative household contacts. The longitudinal nature of data from serial samples will be used to identify household and individual factors independently associated to MRSA colonization and time to MRSA clearance, using the Cox regression model. Associations between time to clearance and independent risk factors will be estimated by means of hazard ratios, with corresponding 95\% CIs. Robust methods will be used to estimate standard errors, to account for dependence of observations within households.

Data on chains of transmission with clonal strains within households will enable estimation of household reproduction numbers, i.e. the average number of colonizations among household contacts arising from an index patient, as well as enabling assessment of MRSA colonization resulting from contact with sources other than household contacts.

Phylogenetic analysis of isolated strains will enable study of the genetic relatedness of MRSA strains associated with hospital encounters and MRSA strains circulating in the community.

\section{Discussion}

As in many other countries, MRSA is endemic in Singapore. Increased efforts at infection control in hospitals 
in recent years have contributed to a reported decline in MRSA burden and subsequent stabilization of disease incidence [30].

Studies of household contacts of MRSA index patients have not been conducted in Singapore, and no longitudinal studies in the community have been done in Southeast Asia. This project is novel since it will be the first to conduct a longitudinal investigation of community transmission of MRSA in Singapore. The study will help determine the direction of transmission and the MRSA carriage dynamics over time. Understanding the dynamics of MRSA persistence and transmission in the community is crucial to devising and evaluating successful MRSA control strategies. Close contact with MRSA colonized patients may be important for MRSA persistence in the community; evidence from this study on the extent of community MRSA could inform the development of household- or community-based interventions to reduce MRSA colonization of close contacts and subsequent reintroduction of MRSA into healthcare settings.

The study will use whole genome sequencing (WGS) to delineate the molecular epidemiology of MRSA within the healthcare system in Singapore. This will help to determine whether these different types of institutions share a common epidemiology dictated by the continuing traffic of MRSA between institutions. MRSA isolates from nasal, axillary and groin (NAG) swab cultures will be typed by WGS and phylogenetic analysis used to establish the relationship between MRSA populations in these different settings. Data from WGS provides superior resolution and is less prone to false-positive and false-negative findings [45]. WGS makes it possible to achieve informative phylogenetic data which can provide additional information about transmission events. Phylogenetic analysis of isolated strains will also enable the study of the genetic relatedness of MRSA strains associated with hospital encounters and MRSA strains circulating in the community.

\section{Abbreviations}

ACH: Acute Care Hospitals; CA-MRSA: Community-associated Methicillin-resistant Staphylococcus aureus; Cl: Confidence Interval; DEFF: Design Effect;

DNA: Deoxyribonucleic Acid; HA-MRSA: Healthcare-associated Methicillin-resistant Staphylococcus aureus; MRSA: Methicillin-resistant Staphylococcus aureus: NAG: Nasal, Axilla, Groin; NEB: New England Biolabs; NHG-DSRB: National Health Group Domain Specific Review Board; PCR: Polymerase Chain Reaction;

ST: Sequence Type; WGS: Whole Genome Sequencing

\section{Acknowledgments}

Not applicable

\section{Funding}

This study is funded by a Communicable Diseases Public Health Research grant from the Ministry of Health, Singapore (grant number: CDPHRG/0010/2014).

Availability of data and materials

Not applicable.
Authors' contributions

NS, CCT and PFdS drafted the manuscript. All authors commented on and approved the final manuscript.

\section{Ethics approval and consent to participate}

Interested patients will be asked to provide signed informed consent and provide consent to contact their household for the purposes of recruiting household members. For participants suffering from cognitive impairment, informed consent will be obtained from their legally appointed representative, usually a spouse or family member responsible for making healthcare decisions on their behalf. For participants between the age of 6 and 12, assent will be obtained from the child and informed consent will be obtained from their legally appointed representative, usually a parent or guardian responsible for making decisions on their behalf.

Ethical approval for this study has been obtained from the National Health Group Domain Specific Review Board (NHG-DSRB 2014/01278).

\section{Consent for publication}

All authors approved the final manuscript.

\section{Competing interests}

The authors declare that they have no competing interests.

\section{Publisher's Note}

Springer Nature remains neutral with regard to jurisdictional claims in published maps and institutional affiliations.

\section{Author details \\ 'Saw Swee Hock School of Public Health, Tahir Foundation Building, 12 Science Drive 2, 11-01, Singapore 117549, Singapore. ${ }^{2}$ Tan Tock Seng Hospital, Singapore, Singapore. ${ }^{3}$ National University Hospital, Singapore, Singapore. ${ }^{4}$ Genome Institute of Singapore, Singapore, Singapore. ${ }^{5}$ London School of Hygiene and Tropical Medicine, London, UK.}

Received: 28 August 2017 Accepted: 4 October 2017

Published online: 11 October 2017

\section{References}

1. Baggett HC, Hennessy TW, Rudolph K, Bruden D, Reasonover A, Parkinson A, J: Community-onset methicillin-resistant Staphylococcus aureus associated with antibiotic use and the cytotoxin Panton-Valentine leukocidin during a furunculosis outbreak in rural Alaska. Dis 2004, 189 SRC - GoogleScholar:1565-1573.

2. Ellis MW, Hospenthal DR, Dooley DP, Gray PJ, Murray CK: Natural history of community-acquired methicillin-resistant Staphylococcus aureus colonization and infection in soldiers. Clin Infect Dis 2004, 39 SRC GoogleScholar:971-979.

3. Frazee BW, Lynn J, Charlebois ED, Lambert L, Lowery D, Perdreau-Remington F: High prevalence of methicillin-resistant Staphylococcus aureus in emergency department skin and soft tissue infections, vol 45; 2005.

4. Kluytmans J, van Belkum A, Verbrugh H. Nasal carriage of Staphylococcus Aureus: epidemiology, underlying mechanisms, and associated risks. Clin Microbiol Rev. 1997;10:505-20.

5. Nakashima AK, Allen JR, Martone WJ, Plikaytis BD, Stover B, Cook LN. Epidemic bullous impetigo in a nursery due to a nasal carrier of Staphylococcus Aureus: role of epidemiology and control measures. Infect Control. 1984;5:326-31.

6. Solberg CO, J.: Spread of Staphylococcus aureus in hospitals: causes and prevention. Scand Dis 2000, 32 SRC - GoogleScholar:587-595.

7. Vandenbergh MF, Verbrugh HA. J.: carriage of Staphylococcus Aureus: epidemiology and clinical relevance. Clin Med. 1999;133:525-34.

8. Wang JT, Chang SC, Ko WJ, Chang YY, Chen ML, Pan HJ, J.: A hospitalacquired outbreak of methicillin-resistant Staphylococcus aureus infection initiated by a surgeon carrier. Infect 2001, 47 SRC - GoogleScholar:104-109.

9. Barnes SL, Harris AD, Golden BL, Wasil EA, Furuno JP. Contribution of Interfacility patient movement to overall methicillin-resistant Staphylococcus Aureus prevalence levels. Infect Control Hosp Epidemiol. 2011;32(11):1073-8.

10. Barr B, Wilcox MH, Brady A, Parnell P, Darby B, Tompkins D. Prevalence of methicillin-resistant Staphylococcus Aureus colonization among older residents of care homes in the United Kingdom. Infect Control Hosp Epidemiol. 2007;28(7 SRC - GoogleScholar):853-9. 
11. Chow A, Lim W, Khan A, Pettigrew K, Lye DCB. Mrsa: dynamics among interconnected acute, intermediate-term, and long-term healthcare facilities in Singapore. Clinical Infectious Diseases 64S2 2017:S76-81. SRC Googlescholar.

12. Donker T, Wallinga J, Slack R, Grundmann H, LOS ONE: Hospital networks and the dispersal of hospital-acquired pathogens by patient transfer. $P$ e35002 2012, 7(4 SRC - GoogleScholar).

13. Gamblin J, Jefferies JM, Harris S, Ahmad N, Marsh P, Faust SN, Fraser S, Moore M, Roderick P, Blair I et al: Nasal self-swabbing for estimating the prevalence of Staphylococcus aureus in the community. Microbiol 62Pt 37 2013:437-440. SRC - GoogleScholar.

14. Garazi M, Edwards B, Caccavale D, Auerbach C, Wolf-Klein G, J.: Nursing homes as reservoirs of MRSA: myth or reality? Med Dir Assoc. 2009, 10 SRC GoogleScholar:414-418.

15. Gorwitz RJ, Kruszon-Moran D, McAllister SK, McQuillan G, McDougal LK, Fosheim GE, Jensen BJ, Killgore G, Tenover FC, Kuehnert MJ, et al. Changes in the prevalence of nasal colonization with Staphylococcus Aureus in the United States. Dis ;:1226-34. 2008;197(9 SRC - GoogleScholar):2001-4.

16. Lee BY, Bartsch SM, Wong KF, Singh A, Avery TR, Kim DS, Brown ST, Murphy CR, Yilmaz SL, Potter MA, et al. The importance of nursing homes in the spread of methicillin-resistant Staphylococcus Aureus (MRSA) among hospitals. Med Care van den The role of nursing homes in the spread of antimicrobial resistance over the healthcare network Infect Control Hosp Epidemiol 377617. 2016;51(3 SRC - GoogleScholar):205-15.

17. van den Dool C, Haenen A, Leenstra T, Wallinga J. The role of nursing homes in the spread of antimicrobial resistance over the healthcare network. Infect Control Hosp Epidemiol. 2016;37(7):761-7.

18. Johansson PJH, Gustafsson EB, Ringberg H: High prevalence of MRSA in household contacts. Scandinavian Journal of Infectious Diseases 9 2007, 39 SRC - GoogleScholar:764-768.

19. Lautenbach E, Tolomeo P, Nachamkin I, Hu B, Zaoutis TE. The impact of household transmission on duration of outpatient colonization with methicillin-resistant Staphylococcus Aureus. Epidemiol Infect. 2010;138(5):638-85.

20. Lucet JC, Paoletti X, Demontpion C, Degrave M. D: Vanjak al Carriage of MethicillinResistant Staphylococcus aureus in Home Care Settings Arch Inern Med. 2009;169(15 SRC - GoogleScholar):1372-8.

21. Mollema FPN, Richardus JH, Behrendt M, Vaessen N, Lodder W: Transmission of Methicillin-Resistant Staphylococcus aureus to Household Contacts. Journal of Clinical Microbiology 1 2010, 48 SRC - GoogleScholar:202-207.

22. Ng W, Faheem A, McGeer A, Simor A, Gelosia A, Willey B, Risk T. Community-and healthcare-associated methicillin-resistant Staphylococcus Aureus strains: an investigation into household and environmental contamination. Infection Control Hospital Epidemiology. 2016;38(01 SRC GoogleScholar):61-7.

23. Knox J, Rijen M, Uhlemann AC, Miller M, C.: Van Hafer al. Communityassociated methicillinresistant Staphylococcus aureus transmission in households of infected cases a pooled analysis of primary data from three studies across international settings Epidemiol Infect 2015, 143 SRC - GoogleScholar:354-365.

24. Rafee Y, Abdel-Haq N, Asmar B, Salimnia T, Pharm CV, C. BM: Increased prevalence of methicillin-resistant Staphylococcus aureus nasal colonization in household contacts of children with community disease. Diseases 45 2012, 12 SRC - GoogleScholar.

25. Nerby JM, Gorwitz R, Lesher L, Juni B, Jawahir S: Risk Factors for Household Transmission of Community-associated Methicillin-resistant Staphylococcus aureus. The Pediatric Infectious Disease Journal 2011, 30 SRC GoogleScholar:927-932.

26. Hsu L, Tan T, Jureen R, Koh T, Krishnan P, Lin R. Antimicrobial drug resistance in Singapore hospitals. Emerg Infect Dis. 2007;13(12):1944-7.

27. Hsu LY, Loomba-Chlebicka N, Koh YL, Tan TY, Krishnan P, Lin RT, Evolving E, J.: 15 epidemic in Singapore hospitals. Microbiol 2007, 56 SRC GoogleScholar:376-379.

28. Hsu LY, Harris SR, Chlebowicz MA, Lindsay JA, Koh TH: Evolutionary dynamics of methicillin-resistant Staphylococcus aureus within a healthcare system. Genome Biology 81 2015, 16 SRC - GoogleScholar.

29. Hsu LY, Koh TH, Tan TY, Ito T, Ma XX, Med J: Emergence of communityassociated methicillin-resistant Staphylococcus aureus in Singapore: a further six cases. Singapore 20 2006, 47(1 SRC - GoogleScholar).

30. Huang YC, Ho CF, Chen CJ, LH S, Lin TY. Nasal carriage of methicillinresistant Staphylococcus Aureus in household contact of children with community-acquired diseases in Taiwan. Pediatr Infect Dis J. 2007;26(11):1066-8.

31. Fisher D, Tambyah PA, Lin RTP. J.: sustained methicillin-resistant Staphylococcus Aureus control in a hyper-endemic tertiary acute care hospital with infrastructure challenges in Singapore. Infection. 2013;85(2 SRC - GoogleScholar):141-8.

32. Pereira LA, Fisher DA. Methicillin-resistant Staphylococcus Aureus control in Singapore: moving forward. Ann Acad Med Singap. 2008;37(10):891-6.

33. Harrison EW, Ludden C, Brodrick HJ, Blane B, Brennan G: Transmission of methicillin-resistant Staphylococcus aureus in long-term care facilities and their related healthcare networks. Genome Med 102 2016, 8 SRC GoogleScholar.

34. Harris SR, Feil EJ, Holden MT, Quail MA, Nickerson EK, Chantratita N. Evolution of MRSA during hospital transmission and intercontinental spread. Science. 2010:327:469-74.

35. Parkhill J, Wren BW: Bacterial epidemiology and biology-lessons from genome sequencing. Genome Biol 230 2011, 12 SRC - GoogleScholar.

36. Price JR, Golubchik T, Cole K. Whole-genome sequencing shows that patient-to-patient transmission rarely accounts for acquisition of Staphylococcus Aureus in an intensive care unit. Clin Infect Dis. 2014;58:609-18.

37. Tong SYC, Holden MTG, Nickerson EK, Cooper BS, Köser CU, Cori A, Jombart T, Cauchemez S, Fraser C, Wuthiekanun V, et al. Genome sequencing defines phylogeny and spread of methicillin-resistant Staphylococcus Aureus in a high transmission setting. Genome Res. 2015;25(1):111-8.

38. Price JR, Gordon NC, Crook D, Llewelyn M, Paul J, Tong SY, Holden MT, Nickerson EK: The usefulness of whole genome sequencing in the management of Staphylococcus aureus infections. Clin Microbiol Infect Genome sequencing defines phylogeny and spread of methicillinresistant Staphylococcus aureus in a high transmission setting Genome Res 251118 2015, 19 SRC - GoogleScholar:784-789.

39. Kong Z, Zhao P, Liu H, Yu X, Qin Y, Su Z, LoS ONE: Whole-Genome Sequencing for the Investigation of a Hospital Outbreak of MRSA in China. P e0149844 2016, 11 (3 SRC - GoogleScholar).

40. Pallen MJ, Loman NJ: Are diagnostic and public health bacteriology ready to become branches of genomic medicine? Genome Med. 53 2011, 3 SRC - Googlescholar.

41. Eyre DW, Golubchik T, Gordon NC, J. BM: A pilot study of rapid benchtop sequencing of Staphylococcus aureus and Clostridium difficile for outbreak detection and surveillance. e001124 2012, 2 SRC - GoogleScholar.

42. Alam MT, Read TD, Petit RA, M.: III, Transmission and microevolution of USA300 MRSA in U. S households evidence from wholegenome sequencing e00054 2015, 6 SRC - GoogleScholar.

43. Popovich KJ, Snitkin E, Green SJ, Aroutcheva A, Hayden MK, Hota B, Weinstein RA. Genomic epidemiology of USA300 methicillin-resistant Staphylococcus Aureus in an Urban Community. Clinical Infectious Diseases: An Official Publication of the Infectious Diseases Society of America. 2016:62(1):37-44.

44. Uhlemann AC, Dordel J, Knox JR, Raven KE, Parkhill J. Molecular tracing of the emergence, diversification and transmission of $\mathrm{S}$ aureus sequence type 8 in a New York community PNAS. 2014;1 11 (18 SRC - GoogleScholar):6738-43.

45. Salipante SJ, SenGupta DJ, Cummings LA, Land TA, Hoogestraat DR, Cookson BT. Application of whole-genome sequencing for bacterial strain typing in molecular epidemiology. J Clin Microbiol. 2015;53(4):1072-9.

\section{Submit your next manuscript to BioMed Central and we will help you at every step:}

- We accept pre-submission inquiries

- Our selector tool helps you to find the most relevant journal

- We provide round the clock customer support

- Convenient online submission

- Thorough peer review

- Inclusion in PubMed and all major indexing services

- Maximum visibility for your research

Submit your manuscript at www.biomedcentral.com/submit 